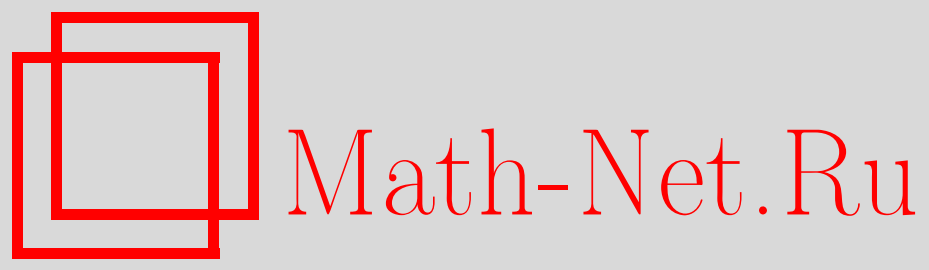

Р. С. Исмагилов, О свойстве локальной коммутативности, Функи. анализ и его прил., 1998, том 32, выпуск 4, 82-85

DOI: https://doi.org/10.4213/faa387

Использование Общероссийского математического портала Math$\mathrm{Net.Ru}$ подразумевает, что вы прочитали и согласны с пользовательским соглашением

http://www . mathnet.ru/rus/agreement

Параметры загрузки:

IP : 52.205 .19 .152

26 апреля 2023 г., 10:18:48

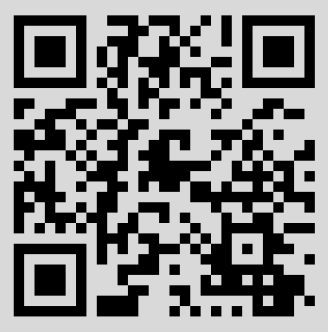




\title{
О свойстве локальной коммутативности
}

\author{
(c) 1998. Р. С. ИСМАГИлОВ
}

1. Постановка задачи. Пусть $C^{\infty}\left(S^{1}\right)$ - пространство вещественных $C^{\infty}$-функций на окружности $S^{1}$. Рассмотрим всевозможные пары $(L, f)$, где $L$ - вещественная алгебра Ли, а $f: C^{\infty}\left(S^{1}\right) \rightarrow L$ - линейное отображение со следующими свойствами: (а) если $u, v \in C^{\infty}\left(S^{1}\right), \operatorname{supp} u \cap \operatorname{supp} v=\varnothing$, то $[f(u), f(v)]=0 ;(б) f\left(C^{\infty}\left(S^{1}\right)\right)$ порождает $L$ как алгебру Ли. Свойство (a) называется локальной коммутативностью [1]. Пару $(L, f)$ будем называть локально коммутативной. Пару $\left(L^{0}, f^{0}\right)$ назовем универсальной, если для любой пары $(L, f)$ существует гомоморфизм алгебр Ли $p: L^{0} \rightarrow L$, такой, что $f=p \circ f^{0}$. На «абстрактном уровне» пара $\left(L^{0}, f^{0}\right)$ описывается так. В тензорной алгебре $A=\bigoplus_{n=1}^{\infty} A_{n}, A_{1}=C^{\infty}\left(S^{1}\right), A_{n}=A_{1}^{\otimes n}$ введем скобку Ли $[u, v]=u \otimes v-v \otimes u$. Возьмем подалгебру Ли $A^{0}=\bigoplus_{n=1}^{\infty} A_{n}^{0}$, $A_{1}^{0}=A_{1}, A_{n}^{0}=\left[A_{n-1}^{0}, A_{1}^{0}\right]$, и ее идеал $I=\bigoplus_{n=2}^{\infty} I_{n}$, порожденный элементами $[u, v], u, v \in A_{1}^{0}, \operatorname{supp} u \cap \operatorname{supp} v=\varnothing$. Тогда алгебра Ли $A^{0} / I$ с очевидным вложением $C^{\infty}\left(S^{1}\right) \rightarrow A^{0} / I$ и есть универсальная локально коммутативная пара. Пространства $A_{n}, n \geqslant 1$, очевидным образом вложены в пространства $C^{\infty}\left(\left(S^{1}\right)^{n}\right)$.

Цель работы - явное (вместо приведенного «абстрактного») описание универсальной пары.

Отметим следующую модификацию этой задачи. Заметим, что $A^{0}=$ $\bigoplus_{n=1}^{\infty} A_{n}^{0}$ есть свободная алгебра Ли над линейным пространством $A_{1}^{0}=$ $C^{\infty}\left(S^{1}\right)$; это означает, что для любой алгебры Ли $B$ и линейного отображения $\varphi: A_{1}^{0} \rightarrow B$ существуют линейные отображения $\varphi^{(n)}: A_{n}^{0} \rightarrow B, \varphi^{(1)}=\varphi$, задающие гомоморфизм алгебр Ли $A^{0} \rightarrow B$. Возьмем целое $p \geqslant 0$ и рассмотрим лишь те локально коммутативные пары $(L, f)$, в которых $L-$ топологическая (локально выпуклая) алгебра Ли, а все отображения $f^{(n)}: A_{n}^{0} \rightarrow L$ непрерывны относительно соболевской нормы

$$
\|u\|_{p}=\max \left\{\left|\prod_{k=1}^{n}\left(\frac{\partial}{\partial x_{k}}\right)^{\alpha_{k}} u\right|, \sum_{k=1}^{n} \alpha_{k} \leqslant p\right\} .
$$

Соответствующую универсальную пару назовем парой порядка $p$; требуется описать ее явно.

В заключение этого раздела опишем подпространства $A_{n}^{0} \subset A_{n}$, используя действие симметрической группы $S_{n},(\sigma u)\left(x_{1}, \ldots, x_{n}\right)=u\left(x_{1 \sigma}, \ldots, x_{n \sigma}\right)$, $\sigma \in S_{n}, u \in A_{n}$. Рассмотрим циклы $t_{k}=(k \cdots 1) \in S_{n}, 1 \leqslant k \leqslant n$, и элементы $p_{k}=\left(1-t_{k}\right) \cdots\left(1-t_{2}\right) \in \mathbb{Z}\left(S_{n}\right), k \geqslant 2, p_{1}=1$. Тогда $A_{n}^{0}=p_{n}\left(A_{n}\right)$. Более того, если определить $\left[f_{1}, \ldots, f_{n}\right]$ формулами $\left[f_{1}, \ldots, f_{n}\right]=\left[\left[f_{1}, \ldots, f_{n-1}\right], f_{n}\right]$, то $\left[f_{1}, \ldots, f_{n}\right]=p_{n}\left(f_{1} \otimes \cdots \otimes f_{n}\right)$. 
2. Результаты. Рассмотрим градуированную алгебру Ли $D=\bigoplus_{n=1}^{\infty} D_{n}$, где $D_{1}=C^{\infty}\left(S^{1}\right)$, а $D_{n}$ при $n \geqslant 2$ есть пространство ростков функций из $A_{n}^{0}$ в окрестности диагонали $x_{1}=\cdots=x_{n}, x_{k} \in S^{1}$. Скобка Ли определяется так же, как в $A^{0}$. Имеем вложение $\varphi: C^{\infty}\left(S^{1}\right)=D_{1} \rightarrow D$.

Теорема 1. $(D, \varphi)$ - универсальная локально коммутативная пара.

Из этой теоремы получается также описание универсальной пары порядка $p \geqslant 0$; ограничимся случаем $p=1$. Рассмотрим алгебру Ли $E=\bigoplus_{n=1}^{\infty} E_{n}$, где $E_{n}=C^{\infty}\left(S^{1}\right),\left[E_{m}, E_{n}\right]=0$ при $m \geqslant 2, n \geqslant 2$, а отображения [•, • ] : $E_{1} \times E_{1} \rightarrow E_{2},[\cdot, \cdot]: E_{1} \times E_{n} \rightarrow E_{n+1}, n \geqslant 2$, имеют вид (соответственно) $[u, v]=u v^{\prime}-u^{\prime} v,[u, v]=u v$. Имеет место вложение $\psi: C^{\infty}\left(S^{1}\right)=E_{1} \rightarrow E$.

ТЕОрема 2. $(E, \psi)$ - универсальная пара порядка 1.

Ядро доказательства теоремы 1 - следующее свойство элементов $t_{k} \in S_{n}$, $p_{n} \in \mathbb{Z}\left(S_{n}\right)$, указанных в разд. 1 .

ТЕОРема 3. При $n \geqslant 2$ суиествует такой элемент $d_{n} \in \mathbb{Z}\left(S_{n}\right)$, что $\operatorname{supp} d_{n} \cap S_{n-1}=\varnothing u p_{n} \cdot\left(1+d_{n}\right)=0$. Точнее, $p_{n} \cdot\left(1+t_{n} p_{n-1}\right)=0, n \geqslant 2$.

(Мы использовали естественное вложение $S_{n-1} \subset S_{n}$ и отождествили элементы из $\mathbb{Z}\left(S_{n}\right)$ с функциями, определенными на $S_{n}$.)

3. Доказательство теорем 1 и 2. Теорема 1 равносильна следующей лемме.

ЛЕмма 1. $I_{n}, n \geqslant 2$, совпадает с множеством функций из $A_{n}^{0}$, равньх нулю в окрестности диагонали $x_{1}=\cdots=x_{n}, x_{k} \in S^{1}$.

ДокАЗАтЕльство. Достаточно рассмотреть случай $n \geqslant 3$. Пусть $d(x, y)$, $x, y \in S^{1}$, - естественная метрика на $S^{1}$. Положим

$$
D(m, n ; \varepsilon)=\left\{\left(x_{1}, \ldots, x_{n}\right) \mid x_{k} \in S^{1}, d\left(x_{i}, x_{k}\right)<\varepsilon \text { при } i \leqslant m, k \leqslant m\right\},
$$

где $0<m \leqslant n, \varepsilon>0$. Пусть функция $u \in A_{n}^{0}$ равна нулю на некотором $D(n, n ; \varepsilon)$; докажем, что $u \in I_{n}$. Как отмечено в разд. $1, u=p_{n}(v)$ для некоторой функции $v \in A_{n}$. Можно считать, что $v=0$ на $D(n, n ; \varepsilon / 2)$ (действительно, возьмем $S_{n}$-инвариантную функцию $\alpha\left(x_{1}, \ldots, x_{n}\right)$, равную нулю на $D(n, n ; \varepsilon / 2)$ и единице вне $D(n, n ; \varepsilon)$; тогда $\left.u=p_{n}(\alpha v)\right)$. Пусть $m$ - наименьшее число, для которого функцию $v$ можно выбрать равной нулю на $D(m, n ; \varepsilon / 2)$. Покажем, что $m=2$. Допустим, что $m>2$. Возьмем функцию $\beta\left(x_{1}, \ldots, x_{n}\right)$, равную единице на $D(m-1, n ; \varepsilon / 4)$ и нулю вне множества $D(m-1, n ; \varepsilon / 2)$. Используя теорему 3 (мы считаем ее доказанной), получаем $u=p_{n}((1-\beta) v+\beta v)=p_{n}\left((1-\beta) v-d_{m}(\beta v)\right)=p_{n}(w)$, где $w=(1-\beta) v+\sum_{\sigma} \lambda(\sigma) \sigma(\beta v) ;$ в последней сумме $\sigma \in S_{m}, \sigma \notin S_{m-1}, \lambda(\sigma) \in \mathbb{Z}$. Функция $\sigma(\beta v)=\sigma(\beta) \sigma(v)$ равна нулю на $\left(\left(S^{1}\right)^{n} \backslash \sigma^{-1}(D(m-1, n ; \varepsilon / 2))\right) \cup$ $D(m, n ; \varepsilon / 2)$. Так как $\sigma \in S_{m}, \sigma \notin S_{m-1}$, то это множество содержит в себе $D(m-1, n ; \varepsilon / 2)$. Итак, $\sigma(\beta v)=0$ на $D(m-1, n ; \varepsilon / 2)$. Поэтому $w=0$ на $D(m-1, n ; \varepsilon / 4)$, что противоречит минимальности числа $m$. Итак, $m=2$. Но тогда функция $u=p_{n}(w)$ лежит в идеале алгебры $A^{0}$, порожденном множеством $I_{2}$. Таким образом, $u \in I_{n}$.

Ясно, с другой стороны, что любая функция из $I_{n}$ равна нулю в окрестности диагонали $x_{1}=\cdots=x_{n}$. Этим доказаны лемма и теорема 1 .

Теорема 2 выводится из теоремы 1 (следует перейти от ростков функций около диагонали $x_{1}=\cdots=x_{n}$ к струям порядка 1$)$. 
4. Доказательство теоремы 3. Число $c \in\{1, \ldots, n\}$ назовем точкой локального минимума элемента $\sigma \in S_{n}, n \geqslant 2$, если $c \sigma<(c-1) \sigma, c \sigma<$ $(c+1) \sigma$; разумеется, если $c=1$ либо $c=n$, то следует взять лишь одно из этих неравенств. Обозначим через $H_{n}$ множество элементов $\sigma \in S_{n}$ с единственной точкой локального минимума $c=M(\sigma)$.

Лемма 2. $H_{n}$ совпадает с множеством элементов $\sigma \in S_{n}$ вида $\sigma=$ $t_{n}^{\varepsilon_{n}} \cdots t_{2}^{\varepsilon_{2}}, \varepsilon_{i} \in\{0,1\}$. Числа $\varepsilon_{i}$ однозначно определяются элементом $\sigma, u$ $M(\sigma)=1+\varepsilon_{2}+\cdots+\varepsilon_{n}$.

Лемма доказывается (несложно) индукцией по $n$.

Пусть $L_{n}$ - множество таких элементов $\psi \in S_{n}$, у которых ровно две точки локального минимума и $1 \psi \neq n, n \psi \neq n$. Положим также

$$
H_{n}^{0}=\left\{\sigma \mid \sigma \in H_{n}, 1<M(\sigma)<n\right\}, \quad H_{n}^{1}=\left\{\tau \mid \tau \in H_{n}, 1 \tau=n\right\} .
$$

Лемма 3. Справедливо равенство $L_{n}=H_{n}^{0} \cdot H_{n}^{1}$. Любой элемент $\psi \in L_{n}$ допускает ровно два разложения вида $\psi=\sigma_{i} \tau_{i}, i=1,2, \sigma_{i} \in H_{n}^{0}, \tau_{i} \in H_{n}^{1}$, причем $M\left(\sigma_{1}\right)=M\left(\sigma_{2}\right),\left|M\left(\tau_{1}\right)-M\left(\tau_{2}\right)\right|=1$.

ДокАЗАТЕльство. Опустим несложное доказательство включения $H_{n}^{0} \cdot H_{n}^{1}$ $\subset L_{n}$. Взяв любой элемент $\psi \in L_{n}$, построим указанные в лемме разложения. Пусть $a$ и $c$ - точки локального минимума для $\psi, b \psi=n$. Ясно, что либо $a \psi=1$, либо $c \psi=1,1 \leqslant a<b<c \leqslant n$. Пусть $c \psi=1$. Положим $F_{1}=$ $\{a, \ldots, c\} \psi, G_{1}=\{1, \ldots, n\} \backslash F_{1}$. Возьмем элемент $\tau_{1} \in S_{n}$, осуществляющий монотонно убывающее отображение множества $\{1, \ldots, c-a+1\}$ на $F_{1}$ и монотонно возрастающее отображение множества $\{c-a+2, \ldots, n\}$ на $G_{1}$. Положим $\sigma_{1}=\psi \tau_{1}^{-1}$. Легко проверить, что $\sigma_{1} \in H_{n}^{0}, \tau_{1} \in H_{n}^{1}, M\left(\sigma_{1}\right)=b$, $M\left(\tau_{1}\right)=c-a+1$. Итак, искомое разложение $\psi=\sigma_{1} \tau_{1}$ построено. Аналогично, из множеств $F_{2}=\{a+1, \ldots, c\} \psi, G_{2}=\{1, \ldots, n\} \backslash F_{2}$ получаем второе разложение $\psi=\sigma_{2} \tau_{2}, \sigma_{2} \in H_{n}^{0}, \tau_{2} \in H_{n}^{1}, M\left(\sigma_{2}\right)=b, M\left(\tau_{2}\right)=c-a$. Легко подсчитать, что $\left|H_{n}^{0}\right|\left|H_{n}^{1}\right|=2\left|L_{n}\right|$. Поэтому таких разложений ровно два.

Докажем теорему 3. Положим $h_{n}=p_{n}\left(1+t_{n} p_{n-1}\right)$. Следует доказать, что $h_{n}=0, n \geqslant 2$. Имеем

$$
h_{n}=p_{n}+\sum(-1)^{\sum_{i=2}^{n} \alpha_{i}} t_{n}^{\alpha_{n}} \cdots t_{2}^{\alpha_{2}} t_{n} p_{n-1}, \quad \alpha_{i} \in\{0,1\} .
$$

Пусть $A_{n}-$ сумма тех слагаемых последней суммы, в которых $0<\sum_{i=2}^{n} \alpha_{i}$ $<n-1$. Тогда

$$
A_{n}=\sum(-1)^{\sum_{i=2}^{n} \alpha_{i}+\sum_{k=2}^{n-1} \beta_{k}} t_{n}^{\alpha_{n}} \cdots t_{2}^{\alpha_{2}} t_{n} t_{n-1}^{\beta_{n-1}} \cdots t_{2}^{\beta_{2}} \quad\left(0<\sum_{i=2}^{n} \alpha_{i}<n-1\right) .
$$

Слагаемое этой суммы можно переписать в виде $(-1)^{M(\sigma)+M(\tau)} \sigma \tau$, где $\sigma=$ $t_{n}^{\alpha_{n}} \cdots t_{2}^{\alpha_{2}}, 0<\sum_{i=2}^{n} \alpha_{i}<n-1, \tau=t_{n} t_{n-1}^{\beta_{n-1}} \cdots t_{2}^{\beta_{2}}$. Согласно леммам 2,3 , $\sigma \tau$ пробегает множество $L_{n}$, и каждый элемент из $L_{n}$ входит в нашу сумму дважды, причем со знаками + и - . Поэтому $A_{n}=0$. Итак, $h_{n}=p_{n}+$ $t_{n} p_{n-1}+(-1)^{n-1} t_{n} \cdots t_{2} t_{n} p_{n-1}=\theta_{n} p_{n-1}$, где $\theta_{n}=1+(-1)^{n-1} t_{n} \cdots t_{2} t_{n}$. Легко проверить, что $\theta_{n}\left(1-t_{n-1}\right)=\left(1-t_{n-1}\right) \theta_{n-1}$, откуда $h_{n}=\left(1-t_{n-1}\right) h_{n-1}$. Отсюда и из очевидного равенства $h_{2}=0$ вытекает, что $h_{n}=0$. Теорема доказана. 
Автор благодарен рецензенту за ценные замечания.

\title{
ЛИТЕРАТУРА
}

1. Nahm W. Quantum field theories in one and two dimensions. Duke Math. J., 54, No. 2, 579-613 (1987).

Московский государственный

технический университет им. Н. Э. Баумана

Поступило в редакцию

11 июня 1997 г.

УДК 517.9

\section{Конструкция канонической следовой плотности деформационного квантования с разделением переменных}

\author{
(C) 1998. А. В. КАРАБЕГОВ
}

В данной заметке мы приводим явную конструкцию канонической следовой плотности (см. [1]) деформационного квантования с разделением переменных на псевдокэлеровом многообразии, введенного в [2].

1. Для данного векторного пространства $V$ назовем формальными векторами элементы пространства $\left.V\left[\nu^{-1}, \nu\right]\right]=\left\{v=\sum_{j \geqslant k} \nu^{j} v_{j} \mid v_{j} \in V, k \in \mathbb{Z}\right\}$ формальных рядов Лорана от параметра $\nu$. Так мы определяем поле формальных чисел $\left.\mathscr{K}=\mathbb{C}\left[\nu^{-1}, \nu\right]\right]$, формальные функции, формы и т. д.

Пусть $M$ есть $2 n$-мерное симплектическое многообразие с симплектической формой $\omega_{-1}$ и соответствующей скобкой Пуассона $\{\cdot, \cdot\}, U-$ произвольное открытое подмножество в $M$ и $\left.\mathscr{F}(U)=C^{\infty}(U)\left[\nu^{-1}, \nu\right]\right], \mathscr{F}_{0}(U)=$ $\left.C_{0}^{\infty}(U)\left[\nu^{-1}, \nu\right]\right]$ - пространства формальных функций на $U$.

Пусть на $\mathscr{F}(M)$ определено ассоциативное, непрерывное в $\nu$-адической топологии $\mathscr{K}$-линейное умножение $\star$ ( $\star$-умножение $)$, заданное на функциях $f, g \in C^{\infty}(M)$ формулой $f \star g=\sum_{r \geqslant 0} \nu^{r} C_{r}(f, g)$, где $C_{r}, r \geqslant 0$, суть билинейные дифференциальные по обоим аргументам операторы, такие, что $C_{0}(f, g)=f g$ и $C_{1}(f, g)-C_{1}(g, f)=i\{f, g\}$. Тогда говорят, что на $M$ задано деформационное квантование (см. [3]).

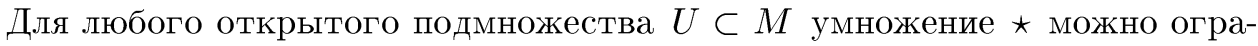
ничить на $U$. Умножение на $\mathscr{F}(U)$ будем обозначать по-прежнему через $\star$.

Следовой плотностью умножения $\star$ на $U$ называется гладкая формальная плотность $d \mu$ на $U$, такая, что для любых $f, g \in \mathscr{F}_{0}(U)$ выполняется соотношение $\int_{U} f \star g d \mu=\int_{U} g \star f d \mu$. Б. В. Федосов построил в [1] глобальную следовую плотность $d \mu_{\text {can }}$ умножения $\star$ на симплектическом многообразии $\left(M, \omega_{-1}\right)$. Эта плотность, называемая канонической, играет важную роль в теоремах об индексе в деформационном квантовании. Старший член формального ряда $d \mu_{\text {саn }}$ равен $(2 \pi \nu)^{-n} \omega_{-1}^{n} / n !$.

Любая следовая плотность $d \mu$ на $U \subset M$ представима в виде $d \mu=\lambda d \mu_{\text {can }}$, где $\lambda$ - локально постоянная формальная функция на $U$. 